\title{
SEMIDERIVATIONS OF PRIME RINGS
}

\author{
MATEJ BREŠAR
}

(Communicated by Maurice Auslander)

\begin{abstract}
A semiderivation of a ring $R$ is an additive mapping $f: R \rightarrow R$ together with a function $g: R \rightarrow R$ such that $f(x y)=f(x) g(y)+x f(y)=$ $f(x) y+g(x) f(y)$ and $f(g(x))=g(f(x))$ for all $x, y \in R$. We prove that the only semiderivations of prime rings are derivations and mappings of the form $f(x)=\lambda(x-g(x))$, where $g$ is an endomorphism and $\lambda$ is an element in the extended centroid.
\end{abstract}

In [2] J. Bergen introduced the notion of a semiderivation: an additive mapping $f$ of a ring $R$ into itself is called a semiderivation if there exists a function $g: R \rightarrow R$ such that

$$
\begin{gathered}
f(x y)=f(x) g(y)+x f(y)=f(x) y+g(x) f(y), \\
f(g(x))=g(f(x)),
\end{gathered}
$$

for all $x, y \in R$. In case $g$ is the identity map on $R, f$ is of course a derivation. The other motivating examples are mappings of the form $f=1-g$, where $g$ is an endomorphism and 1 is the identity map. Recently H. E. Bell and W. S. Martindale showed that if a semiderivation $f$ of a prime ring $R$ satisfies certain conditions then there exists $\lambda$ in the extended centroid of $R$ such that $f(x)=\lambda(1-g)(x)$ for all $x \in R[1$; Theorem 1, Lemma 4, Lemma 5]. The purpose of this note is to prove that the mappings of this form and derivations are in fact the only examples of semiderivations in prime rings. For the proof of this result we need the following lemma which is of independent interest.

Lemma. Let $R$ be a prime ring. If functions $F: R \rightarrow R$ and $G: R \rightarrow R$ are such that $F(x) y G(z)=G(x) y F(z)$ for all $x, y, z \in R$, and $F \neq 0$, then there exists $\lambda$ in the extended centroid of $R$ such that $G(x)=\lambda F(x)$ for all $x \in R$. Proof. Given $x \in R$, for all $y \in R$ we then have $F(x) y G(x)=G(x) y F(x)$; if $F(x) \neq 0$, using a well-known result of Martindale [5; Theorem 1] we have that $G(x)=\lambda(x) F(x)$ for some $\lambda(x)$ in the extended centroid of $R$. Hence, if $x$,

Received by the editors April 7, 1989.

1980 Mathematics Subject Classification (1985 Revision). Primary 16A72, 16A12.

Key words and phrases. Semiderivation, derivation, endomorphism, prime ring, extended centroid. 
$z \in R$ are such that $F(x) \neq 0$ and $F(z) \neq 0$, then the relation $F(x) y G(z)=$ $G(x) y F(z)$ can be written in the form $(\lambda(z)-\lambda(x)) F(x) y F(z)=0$. Since $R$ is prime we are forced to conclude that $\lambda(x)=\lambda(z)$. Thus there exists $\lambda$ in the extended centroid of $R$ such that $G(x)=\lambda F(x)$ is fulfilled for every $x \in R$ which satisfies $F(x) \neq 0$; however, if $F(x)=0$, then we see from $F(x) y G(z)=G(x) y F(z)$, since $F \neq 0$ and $R$ is prime, that $G(x)=0$ too. Therefore $G(x)=\lambda F(x)$ holds for all $x \in R$.

In our forthcoming paper [3] the above lemma is proved for the special case when $F$ and $G$ are derivations.

We are now ready to prove

Theorem. Let $R$ be a prime ring. If $f$ is a semiderivation of $R$ (with associated function $g$ ) then either $f$ is a derivation or $f(x)=\lambda(1-g)(x)$ for all $x \in R$, where $\lambda$ is an element in the extended centroid of $R$ and $g$ is an endomorphism. Proof. We may assume that $f \neq 0$. In this case $g$ is a ring endomorphism [4; Theorem 1]. Note that (1) can be written in the form

$$
(1-g)(x) f(y)=f(x)(1-g)(y) \quad \text { for all } x, y \in R .
$$

In particular, $(1-g)(x) f(y z)=f(x)(1-g)(y z)$ for all $x, y, z \in R$. But on the other hand, $(1-g)(x) f(y z)=(1-g)(x) f(y) g(z)+(1-g)(x) y f(z)$ and $f(x)(1-g)(y z)=f(x)(1-g)(y) g(z)+f(x) y(1-g)(z)$. Comparing the last two relations and applying $(1-g)(x) f(y)=f(x)(1-g)(y)$, we then get $(1-g)(x) y f(z)=f(x) y(1-g)(z)$ for all $x, y, z \in R$. If $g=1$ then $f$ is a derivation; therefore we may assume that $1-g \neq 0$ and so the assertion of the theorem follows immediately from the lemma.

\section{REFERENCES}

1. H. E. Bell and W. S. Martindale, Semiderivations and commutativity in prime rings, Canad. Math. Bull. 31 (1988), 500-508.

2. J. Bergen, Derivations in prime rings, Canad. Math. Bull. 26 (1983), 267-270.

3. M. Brešar, Centralizing mappings and derivations in prime rings, preprint.

4. J. C. Chang, On semiderivations of prime rings, Chinese J. Math. 12 (1984), 255-262.

5. W. S. Martindale, Prime rings satisfying a generalized polynomial identity, J. Algebra 12 (1969), 576-584.

Institute of Mathematics, Physics and Mechanics, University of Ljubljana, P. O. Box 543, 61111 LuUbluana, Yugoslavia 